\title{
Hemocultivos en el Servicio de Pediatría del Instituto Oncológico Nacional "Dr. Juan Tanca Marengo", Solca-Guayaquil.
}

\section{Blood cultures in the Pediatric Service of the "Dr. Juan Tanca Marengo", National Oncology Institulte of Solca-Guayaquill.}

\author{
Luis Espín Custodio1* iD , Aníbal Bonilla Nuñez', Jéssica Andrade \\ Rada1
}

1. Servicio de Oncología Pediátrica. SOLCA - Guayaquil.

*Correspondencia:

lespin@solca.med.ec

Teléfono [593] 998387404

Conflicto de intereses: Los autores declaran no tener conflictos de intereses.

Fondos: Ver la página 124

Recibido: 13 Marzo 2019

Aceptado: 22 Julio 2019

Publicado: 30 Agosto 2019

Membrete bibliográfico:

Espín L, Bonilla A, Andrade J. Hemocultivos en el Servicio de Pediatría del Instituto Oncológico Nacional "Dr. Juan Tanca Marengo", Solca-Guayaquil. Rev. Oncol. Ecu 2019;29(2):119-126.

ISSN: 2661-6653

DOI: https://doi.org/10.33821/87

Copyright Espín et al. Este artículo es distribuido bajo los términos de Creative Commons Attribution License 4.0 , el cual permite el uso y redistribución citando la fuente y al autor original.

\section{Resumen}

Introducción: La creciente resistencia antibiótica de los microorganismos aislados en cultivos de sangre en pacientes neutropénicos hace necesaria la continua monitorización del antibiograma para vigilar la cambiante susceptibilidad antibiótica. El objetivo de este estudio fue identificar a los microorganismos aislados de hemocultivos y su sensibilidad, en niños con cáncer ingresados en un Instituto Oncológico.

Métodos: El presente es un estudio prospectivo de datos recolectados de junio 2017 a junio 2018, con aislamiento de cultivos de sangre en pacientes neutropénicos febriles hospitalizados en el área de pediatría del Instituto Oncológico Nacional “Dr. Juan Tanca Marengo" Solca, Guayaquil.

Resultados: Fueron 183 pacientes, $133(72 \%)$ con neoplasias hematológicas y $50(28 \%)$ con tumores sólidos. Se tomaron 265 hemocultivos. 100 reportes (38\%) fueron positivos, $66 \%$ fueron bacterias Gram-Positivas y $34 \%$ Gram- negativas. Las bacterias más frecuentes $20 \% \mathrm{E}$. epidermidis, $18 \%$ E. aureus, $17 \%$ E. haemolyticus, $12 \%$ E. Coli. Las bacterias Gram-positivas fueron $100 \%$ sensibles al linezolid, vancomicina y tigacilina. Las Gram-negativas mantienen una sensibilidad de $91 \%$ a la amikacina, $59 \%$ a cefepime, $59 \%$ a ceftazidima, $77 \%$ a ciprofloxacina, $68 \%$ a imipenem, $76 \%$ a meropenem, $56 \%$ a piperacilina- tazobactam, $100 \%$ al colistin y $97 \%$ a la tigeciclina. La Klebsiella Pneumoniae productora de Carbapenemasa (KPC) multirresistente fue sensible $100 \%$ a tigaciclina y colistin y $50 \%$ a la amikacina.

Conclusión: La incidencia fue mayor para Gram- positivos. Existe una buena sensibilidad a la vancomicina, linezolid y tigaciclina para bacterias Gram- positivas; y a la amikacina, colistin y tigacilina para las Gram- negativas. Las bacterias KPC multiresistentes fueron solamente sensibles a tigeciclinay colistin.

Palabras Claves: CULTIVO DE SANGRE, HOSPITALES PEDIÁTRICOS, NEUTROPENIA, NEUTROPENIA, BACTERIAS MULTI- RESISTENTES, INFECCIONES BACTERIANAS Y MICOSIS, BACTERIAS AEROBIAS GRAMNEGATIVAS, BACTERIAS GRAMPOSITIVAS. 
DOI: https://doi.org/10.33821/87

\begin{abstract}
Introduction: The increasing antibiotic resistance of isolated microorganisms in blood cultures in neutropenic patients makes it necessary to continuously monitor the antibiogram to monitor the changing antibiotic susceptibility. The objective of this study was to identify during one year the isolated microorganisms of blood cultures and their sensitivity, in children with cancer admitted to an Oncological Institute.
\end{abstract}

Methods: This is a prospective study of data collected from June 2017 to June 2018, with isolation of blood cultures in febrile neutropenic patients hospitalized in the pediatric area of the National OncologyInstitute "Dr. Juan Tanca Marengo "Solca, Guayaquil.

Results: There were 183 patients, 133 (72\%) with hematological malignancies and 50 (28\%) with solid tumors. In this group 265 blood cultures were taken. 100 reports (38\%) were positive, 66\% were Gram- Positive bacteria and 34\% Gram-negative. The most frequent bacteria 20\% E. epidermidis, 18\% E. aureus, $17 \%$ E. haemolyticus, 12\% E. Coli, 10\% Staphylococcus hominis, 9\% Klebsiella Pneumoniae, 6\% Pseudomona species, 5\% Acinetobacter, Gram bacteria -positives were 100\% sensitive to linezolid, vancomycin and tigacillin; while Gram-negatives maintain a sensitivity of $91 \%$ to amikacin, $59 \%$ to cefepime, $59 \%$ to ceftazidime, $77 \%$ to ciprofloxacin, $68 \%$ to imipenem, $76 \%$ to meropenem, $56 \%$ to piperacillin-tazobactam, $100 \%$ at colistin and $97 \%$ at tigecycline. An increase in multi-resistant Carbapenemase-producing Klebsiella Pneumoniae (KPC) was observed, 100\% sensitive to tigacycline and colistin and $50 \%$ to amikacin.

Conclusion: The incidence was higher for gram-positive bacteria. There is a good sensitivity to vancomycin, linezolid and tigacycline for gram-positive bacteria; and to amikacin, colistin and tigacillin for Gram-negatives. The multi-resistant KPC bacteria were only sensitive to tigecycline and colistin.

Keywords: BLOOD CULTURE, PEDIATRIC HOSPITALS, NEUTROPENIA, PEDIATRIC, MULTIRESISTANT BACTERIA, BACTERIAL INFECTIONS AND MICOSIS, GRAMNEGATIVE BACTERIA, GRAMPOSITIVE BACTERIA.

\section{Introducción}

La neutropenia febril es una complicación del tratamiento intenso en el cáncer pediátrico, necesitando del pronto inicio de antibióticos de amplio espectro. Los pacientes de alto riesgo deben ser hospitalizados y recibir terapia antibiótica intravenosa que incluya cobertura para Pseudomona aeruginosa. El tratamiento empírico deberá ser modificado de acuerdo al resultado de los cultivos y la situación clínica [1]. Dada la variabilidad en el tiempo de los microorganismos aislados en los cultivos, se hace necesaria la monitorización continua de los gérmenes a fin de guiar la terapia empírica inicial [2]. En la década de los 70, las infecciones por bacilos Gram- negativos (BGN) causaban $60-70 \%$ de las bacteriemias en pacientes neutropénicos. E.coli, Klebsiella y Pseudomona daban cuenta de la mayoría de episodios. En las décadas siguientes se produjo un ascenso relativo de las infecciones por bacterias Gram-positivas (BGP) [3]. 
En los últimos años, la literatura médica internacional reporta en pacientes pediátricos neutropénicos, la resistencia creciente de los microorganismos a los antimicrobianos comúnmente utilizados $[3,4]$.

El objetivo de este estudio fue identificar durante el período de un año los microorganismos aislados del torrente sanguíneo y su perfil de susceptibilidad, en niños con cáncer ingresados en el área de Hospitalización pediátrica del ION SOLCA Guayaquil, a fin de lograr un esquema empírico inicial efectivo en el manejo de la neutropenia febril.

\section{Materiales y Métodos}

El presente estudio es prospectivo de 12 meses comprendido entre junio del 2017 a junio del 2018. Ingresaron al estudio los pacientes en quienes se solicitó un hemocultivo y/o un retrocultivo. Los participantes fueron pacientes menores de 18 años, ingresados con neoplasias hematológicas y no hematológicas, en el área de Pediatría del Instituto Oncológico Nacional "Dr. Juan Tanca Marengo", de Solca-Guayaquil. Las variables fueron la identificación del patógeno y su sensibilidad. Los hemocultivos fueron tomados directamente de sangre periférica y los retrocultivos de vías venosas centrales o implantofix.

Los hemocultivos fueron sometidos al Sistema BACTEC 9050, identificados por el Sistema API 20 E para Enterobacterias y API 20 NF para bacterias no fermentadoras (NF), y finalmenteel antibiograma por el Sistema ATB.

La recolección de datos se realizó a través de hojas estadísticas y se efectuó la tabulación de cultivos positivos identificando los gérmenes y sus sensibilidades, se utiliza estadística descriptiva. Se obtuvo el permiso para la investigación por parte del Departamento de Investigación del Instituto.

\section{Resultados}

Durante el período de estudio ingresaron a Pediatría 183 pacientes, de los cuales 133 (72 $\%)$ tenían neoplasias hematológicas y 50 (28\%) tumores sólidos. De las neoplasias hematológicas, 116 (63\%) fueron leucemias; 96 (83\%) leucemias linfoblasticas agudas, 20 casos (17\%) fueron leucemias mieloides agudas, 17 casos (9\%) fueron linfomas, 7 casos fueron Linfomas no Hodgkin, 10 casos linfoma Hodgkin.

En los 183 pacientes ingresados, se tomaron 265 cultivos, (78\% hemocultivos y $22 \%$ retrocultivos). 100 reportes (38\%) fueron positivos. De los cultivos positivos, $66 \%$ fueron bacterias Gram- positivas y $34 \%$ Gram- negativas.

De los 100 cultivos positivos aislados: 20 correspondieron a Estafilococo epidermidis, 18 Estafilococo aureus, 17 Estafilococo haemolyticus, 12 E. Coli, 10 Estafilococo hominis, 9

Klebsiella Pneumoniae, 6 Pseudomona species, 5 Acinetobacter, 1 Serratia, 1 Estafilococo saprofiticus y 1 Burkhloderia. 
Sensibilidad a los Antibióticos

El Estafilococo aureus fue sensible a la ciprofloxacina, gentamicina, levofloxacina, linezolid, moxifloxacina, tigacilina, trimetropin sulfametoxazol y vancomicina en $100 \%$ y a eritromicina, clindamicina y oxacilina en $45 \%$. Entre los estafilococos coagulasa negativos (estafilococos no aureus) la sensibilidad a los antibióticos fue: ciprofloxacina $57 \%$, clindamicina $35 \%$, gentamicina $85 \%$, levofloxacina $75 \%$, rifampicina $79 \%$, trimetropin sulfametoxazol $75 \%$, eritromicina $19 \%$, oxacilina $9 \%$, y $100 \%$ de sensibilidad al linezolid, tigacilina, vancomicina y moxifloxacina.

En cuanto a la sensibilidad de las bacterias Gram- negativas: amikacina 91\%, cefepime $59 \%$, ceftazidima 59\%, ciprofloxacina $77 \%$, imipenem $68 \%$, meropenem $76 \%$, piperacilina-

tazobactam $56 \%$, tigaciclina $97 \%$ y colistin $100 \%$.

Figura 1. Resistencia antibiótica de Klebsiella pneumoniae BLEE positivo

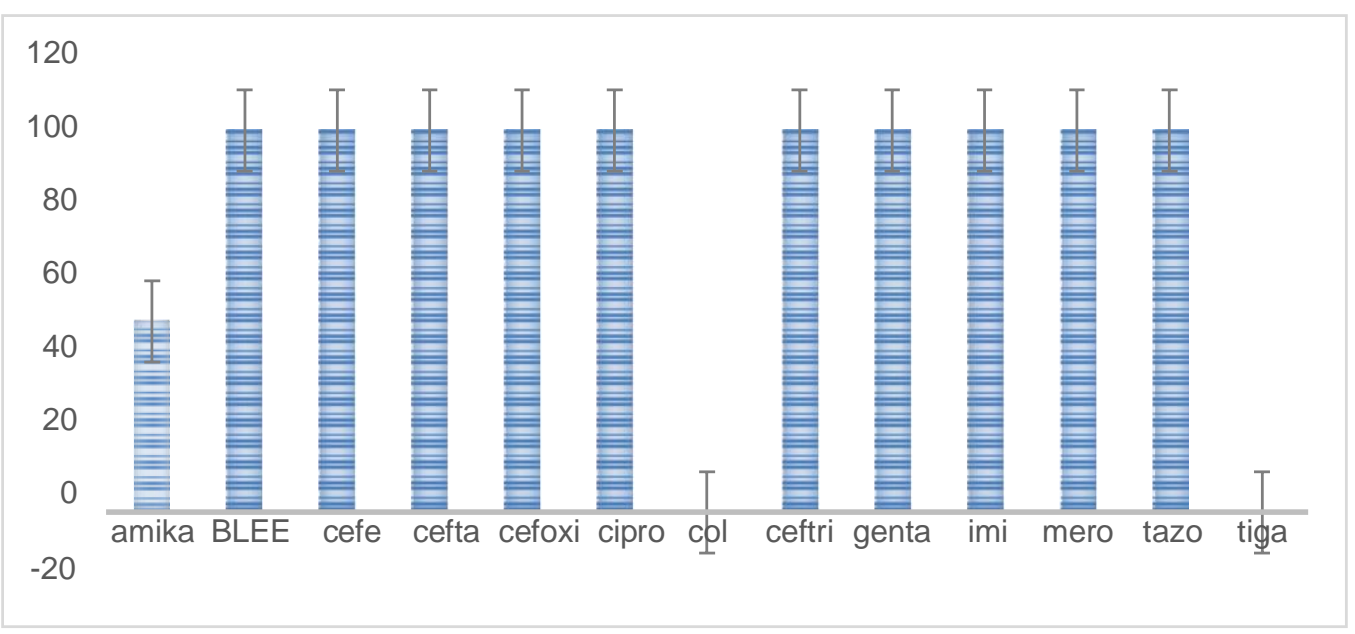

Amika: amikacina, BLEE: Beta Lactamasa Espectro Extendido; cefe: cefepime; cefta: ceftazidima; cefoxi: cefoxitina; cipro: ciprofloxacina; col: colistina; ceftri: ceftriaxona; genta: gentamicina; imi: imipenem; mero: meropenem; tazo: tazobactam; tiga:tigaciciclina.

\section{Discusión}

De los 183 pacientes en el estudio, se obtuvieron 100 cultivos positivos con su respectivo antibiograma. La incidencia fue mayor para los Gram- positivos (66 \%), lo cual distingue a la década del '80 en que se reportaba un predominio de infecciones causadas por bacilos Gram-negativos [5, 6]. El cambio en la epidemiología en pacientes con neutropenia febril, se debe probablemente a la modificación en los tratamientos quimioterapéuticos con manejos más mieloablativos que llevan a mayor intensidad y duración de la neutropenia, aumento del tiempo de internación y por consiguiente, incremento de las infecciones por gérmenes nosocomiales [7, 8].

El gérmen Gram- positivo más frecuente fue el Estafilococo aureus, datos que semejan a estudios previos donde $61 \%$ fueron E. aureus y $51 \%$ E. coagulasa negativos [9]. Entre las 
bacterias Gram- negativas, el estudio mencionado, a diferencia de nuestra mayor incidencia de E. Coli (35\%), reporta a la Pseudomona spp. y Acinetobacter spp. como los más frecuentes y la E. coli solo en $16 \%$ [10]. Comparados con un estudio americano, concordamos con el predominio de E. Coli (51.3\%) entre los agentes aislados en pacientes neutropénicos febriles, seguido de Pseudomona en un $20.1 \%$ [11].

No se presentó ningún caso de candidemia durante el período estudiado lo cual se distingue de datos que indican que las infecciones fúngicas han aumentado su frecuencia durante la última década en niños con NF [12]. C. albicans, C. parapsilosis, C. tropicalis fueron las prevalentes, seguido de Aspergillus spp [13]. En Chile, sobre 445 episodios de $\mathrm{NF}, 9 \%$ presentó diagnóstico de enfermedad fúngica invasora (EFI) prevaleciendo las especies de Cándidas [14]; mientras que en Argentina establecen un $50 \%$ de las candidemias causadas por C. albicans y $17 \%$ por C. parapsilosis [15]. Creemos que la falta de aislamiento de cándidas en nuestro trabajo se debe al uso temprano de fluconazol, anfotericina o caspofungin, aún sin aislamiento del germen, tomando como parámetros la persistencia de la fiebre y la neutropenia mayor a 5 días junto a las imágenes.

Teniendo como referencia estudios previos realizados en ION SOLCA, la sensibilidad de las bacterias Gram- positivas a la vancomicina, linezolid y tigaciclina se ha mantenido en $100 \%$ durante un período de 10 años. La sensibilidad a la clindamicina y oxacilina ha ido disminuyendo paulatinamente. En cuanto a la sensibilidad de las bacterias Gramnegativas en nuestro estudio destaca una buena sensibilidad a la amikacina con un $91 \%$, colistin 100

$\%$ y tigacilina $97 \%$. Ha disminuido al imipenem $68 \%$ y al meropenem $76 \%$ que en el 2014 era de $99 \%$. La sensibilidad a las cefalosporinas de tercera y cuarta generación se mantiene para el cefepime $56 \%$ (58 \% en el 2014) y ceftriaxona $56 \%$ (55\% en 2014), pero ha disminuido para la ceftazidima de $71 \%$ a $59 \%$. La sensibilidad, disminuye o se incrementa si se incluyen o no cultivos KPC positivos, respectivamente. La tigaciclina y colistina se mantienen con las menores tasas de resistencia [16-18]. Llama la atención en nuestro estudio la presencia de bacterias KPC multiresistentes, sensibles $100 \%$ solamente a tigeciclina y colistin y $50 \%$ a la amikacina. Existen numerosos reportes en la literatura médica que describen la utilidad in vitro de tigeciclina contra microorganismos multiresistentes [19], incluyendo K. pneumoniae productora de carbapenemasas (Kpn KPC) [20, 21]. Así mismo fue demostrado en un estudio chileno que concluye que la tigeciclina puede ser efectivo para el tratamiento de infecciones por bacterias multi-resistentes en niños [22].

El esquema de tratamiento inicial empírico usado en este Instituto en base a cefepime con o sin amikacina; piperacilina- tazobactam con o sin amikacina; progresando según evolución a carbapenémicos, hasta ahora ha sido satisfactorio, pero cada vez se hace necesario el empleo de antibióticos como la tigaciclina y el colistin. El empleo de la vancomicina se hace en casos específicos y al inicio del manejo de sepsis. Nuevos estudios deberán valorar la eficacia de estos antibióticos en el futuro. 


\section{Conclusiones}

La sensibilidad de los cultivos de sangre fue del $38 \%$, la mayoría fueron bacterias Grampositivas, de las cuales el Estafilococo aureus es probablemente el único germen patógeno. Se afirma también como en estudios previos, que la elevada frecuencia de estafilococos coagulasa negativos se debe al incremento en el uso de dispositivos intravasculares de larga duración, intensidad de la neutropenia causada por la quimioterapia y uso de profilaxis antibiótica para bacterias Gram- negativas.

\section{Agradecimientos}

Se reconoce a las personas que participaron indirectamente en el estudio tales como los pacientes, como personal técnico, otras en general de ION "Dr. Juan Tanca Marengo" SOLCA-Guayaquil.

\section{Información adicional}

\section{Abreviaturas}

BLEE: Beta Lactamasa Espectro Extendido

KPC: Klebsiella pneumoniae

carbapenemasaNF: Bacterias No

Fermentadoras

EFI: enfermedad Fúngica invasora

ION: Instituto Oncológico Nacional "Dr. Juan Tanca Marengo"

SOLCA: Sociedad de Lucha contra el Cáncer.

Nota del Editor

La Revista Oncología Ecu permanece neutral con respecto a los reclamos jurisdiccionales en mapas publicados $y$ afiliaciones institucionales.

\section{Archivos Adicionales}

Ninguno declarado por los autores.

\section{Fondos}

Los fondos para la presente investigación fueron propios de los autores del presente artículo.

\section{Disponibilidad de datos y materiales}

Existe la disponibilidad de datos bajo solicitud al autor de correspondencia. No se reportan otros materiales. 


\section{Contribuciones de los autores}

LEC, ABN, JAR contribuyeron igual en el proceso de la investigación, idea de investigación, diseño, recolección de datos, análisis estadístico, escritura académica. El análisis crítico del artículo lo realizó LEC y ABN. Todos los autores leyeron y aprobaron la versión final del manuscrito.

Aprobación de ética y consentimiento para participar

No aplica ya que es un estudio observacional retrospectivo.

\section{Consentimiento para publicación}

No aplica ya que es un estudio observacional retrospectivo.

\section{Información de los autores}

Luis Espin Custodio iD, Jefe del Servicio de Pediatría del Instituto Oncológico Nacional "Dr. Juan Tanca Marengo", Solca-Guayaquil. E-mail: lespin@solca.med.ec https://orcid.org/0000-0003-0880-2377

Anibal Bonilla Nuñez, Médico tratante del Servicio de Pediatría del Instituto Oncológico Nacional "Dr. Juan Tanca Marengo", Solca-Guayaquil. E-mail: abonilla@solca.med.ec

Jéssica Andrade Rada, Médica residente del Servicio de Pediatría del Instituto Oncológico Nacional "Dr. Juan Tanca Marengo", Solca-Guayaquil. E-mail: jessicandraderada@gmail.com

\section{Referencias}

1. Kebudi $\mathrm{R}$, Kizilocak $\mathrm{H}$. Febrile neutropenia in children with cancer: Approach to diagnosis and treatment. Curr Pediatr Rev. 2018;14(3):204-209. DOI: $10.2174 / 1573396314666180508121625$

2. Solis $Y$, Alvarez A, Fuentes D, De la Barra D, Aviles C, et al. Agentes causantes de bacteriemia en niños con cancer y neutropenia febril de alto riesgo en seis hospitales de Santiago. Rev Chile Infect. 2012;29(2):156-62. DOI: $10.4067 / S 0716-10182012000200006$

3. Rose W, Veeraraghava B, Pragasam A, Verghese V. Antimicrobial susceptibility profile of isolate from pediatric blood stream infections. Indian Pediatr. 2014;51(9):752-3. PMID:25228617

4. Oliveira AL, De-Souza M, Carvalho Diaz VM. Epidemiology of bacteremia and factors associated with multi drug resistant gramnegative bacteremia in hematopoietic stem cell transplan recipients. Bone Marrow Transplant. 2007;39(12):775-81. DOI: 10.1038/sj.bmt.1705677

5. Özdemir ZC, Koç A, Ayçiçek A. Microorganisms isolated from cultures and infection focus and antibiotic treatments in febrile neutropenic children from Sanliurfa, Turkey. Turkish J Pediatr. 2016;58(1):47-53. PMID: 27922236 
6. Freifeld AG, Bow EJ, Sepkowitz KA, Boeckh MJ, Ito JI, Mullen CA, et al from: Infectious Diseases Society of America. Clinical practice guideline for the use of antimicrobial agents in neutropenic patients with cancer: 2010 update by the infectious diseases society of america. Clin Infect Dis. 2011;52(4):e56-93. DOI: 10.1093/cid/cir073

7. Santolaya M, Rabagliati R, Bidart T, Payá E, Guzmán A, Morales R, et al. Consenso Manejo racional del paciente con cáncer, neutropenia y fiebre. Rev. Chil Infect. 2005;22(Supl 2):S79-S113. DOI: 10.4067/S071610182005000500001

8. Oguz A, Karadeniz C, Citak EC, Cil V, Eldes N. Experience with cefepime versus meropenem as empiric monotherapy for neutropenia and fever in pediatric patients with solid tumors. Pediatr Hematol Oncol. 2006;23(3):245-253. DOI: $10.1080 / 08880010500506867$

9. Freifeld A, Bow EJ, Sepkowitz K, Boeckh MJ, Ito JI, Mullen CA, et al. Clinical practice guideline for the use of antimicrobial agents in neutropenic patients with cancer: 2010 update by the infectious diseases society of america. Clin Infect Dis. 201;52(4):e56-93. DOI: 10.1093/cid/cir073

10. Kobayashi S, Ito M, Sano H, Mochizuki K, Akaihata M, Waragai T, et al. Clinical analysis of combination therapy for febrile neutropenic patients in childhood cancer. Pediatric Int. 2013;55(1):65-71. DOI: $\underline{10.1111 / \text { ped. } 12025}$

11. Trecarichi E, Tumbarello M. Antimicrobial- resistant Gram-negative bacteria in febrile neutropenic patients with cancer: current epidemiology and clinical impact. Curr Opin Infect Dis. 2014;27(2):200-210. DOI: $\underline{10.1097 / Q C O .0000000000000038}$

12. Lucero $\mathrm{Y}$, Brucher R, Alvarez A, Becker A, Cofre J, Enriquez N, et al. Infección micótica profunda en niños con cáncer, neutropenia y fiebre, en Chile. Rev Med Chile. 2002;130:1139-1146. DOI: 10.4067/S0034$\underline{98872002001000008}$

13. Boilee G, Safari C, Thiery G, Bergeron A, de Miranda S, Menotii J, et al. Clinical Picture of Pneumocystis jiroveci pneumonia in cancer patients. Chest. 2007;132(4):1305-1310. PMID:17934116.

14. Ramphal R, Grant R, Dzolganovski B, Constantin J, Telier R, Alien U, et al. Herpes simplex virus in febrile neutropenic children undergoing chemotherapy for cancer: a prospective cohort study. Pediatr Infect Dis J. 2007;26(8):700-704. DOI: 10.1097/INF.0b013e31805cdc11

15. Täger F, Zolezzi R, Folatre B, Navarrete $C$, Rojas $P$. Respiratory virus infections in children with acute lymphoblastic leukemia and febrile neutropenia; a prospective study. Rev Chil Infectol. 2006;23(2):118-123. PMID: 16721444

16. Bonilla A. Perfil microbiológico de infecciones en pediatría SOLCA Guayaquil. Revista Oncología SOLCA. 2005. 15: 135-139.

17. Bonilla A. Perfil microbiológico de infecciones en pediatría SOLCA Guayaquil. Revista Oncología SOLCA. 2001. 11: 191-199

Abreviaturas en la referencias

\section{DOI: Digital Object}

Identifier

PMID: PubMed Identifier

SU: Short URL
18. Bonilla A, Alcívar D, Fuentes H. Hemocultivos en pediatría Instituto Oncológico Nacional (ION) SOLCA. 2014.

19. Chambers H. Protein synthesis inhibitors and miscellaneous antibacterial agents. The Pharmacological Basis of Therapeutics. Chapter 41. From: Hardman JG, Limbird LE, Gilman AG . Goodman \& Gilman's The Pharmacological Basis of Therapeutics (13 ed). Nueva York: McGraw-Hill. 2006. 11: 1095-110. ISBN: 0071354697.

20. Ko K S, Song J H, Lee M Y, Park S, Kwon K T, Heo S T, et al. Antimicrobial activity of tygecicline against recent isolates of respiratory pathogens from Asian countries. Diagn Microbiol Infect Dis 2006;55(4):337-341. DOI: 10.1016/i.diagmicrobio.2006.02.001

21- Betriu C, Rodríguez-Avial I, Gómez M, Culebras E, López F, Álvarez J, et al. Antimicrobial activity of tygecicline against clinical isolates from Spanish medical centers. Second multicenter study. Diagn Microbiol Infect Dis. 2006;56(4):437-444. DOI: 10.1016/j.diagmicrobio.2006.07.005

22. Hurtado I, Trujillo M, Restrepo A, Garcer C, Tamayo C, Mesa J. Experiencia con el uso compasivo de tigeciclina en pacientes pediátricos infectados por Klebsiella pneumoniae productora de carbapenemasas. Rev. Chil. Infectol. 2012;29(3):313-321. DOI: 10.4067/S0716-10182012000300011 